\title{
Appanti per servire alla biografia di Chiaro Davanzati.
}

Di qualche decennio più antica che il Gamurrini e il Bindi 1 non la credano è la famiglia fiorentina dei Davanzati, le cui origini debbono riportarsi almeno alla fine del sec. XII; poichè, se è vero che non prima della battaglia di Montaperti essa incomincia a figurare nella storia del Comune, tuttavia appare già ricordata in atti privati di data anteriore.

Risulta infatti dagli spogli dell' Ancisa conservati nel R. Archivio di stato fiorentino che nel 123 I Riccomanno, Bonello, Jacobo e Adimario fratres filiqque Davanzati vendettero alcune loro terre; ${ }^{2}$ a chi, dove e come noi non lo sappiamo bene, ma ci importa soltanto potere accertare che in quell' anno viveva un Davanzato la cui nascita va certamente riportata alla fine del secolo precedente, se nel 1231 era già padre di quattro figliuoli in età da poter vendere terre.

Più antica testimonianza le mie ricerche non mi hanno offerto: scendendo con gli anni, Davanzato è nuovamente nel 1246, nelle matricole dei mercanti; 3 nel 1256 si fa menzione di Jacobo suo figlio; ${ }^{4}$ infine nel 1260 , l' anno famoso di Montaperti, appaiono nel Libro anonimo che da quella battaglia si intitola, come Pavesari del sesto d' Oltrarno, un Clarus f. Davanzati Banbakai populi Sanctz Fridiani e un Chiarus $f$. Davanzati piopuli Sancte Marie sopr'Arno. ${ }^{5}$

A quale dei due combattenti spetterd l' alloro del poeta? Ecco il dubbio che mi propongo di risolvere.

In favore di Chiaro del popolo di Santa Maria si pronunziò risolutamente il Novati, pubblicando nel 1885 alcuni documenti

1 Le opere di Bernardo Davanzati per cura di Enrico Bindi, Firenze, Le Monnier, 1853. - Gamurrini, Istoria Genealogica delle famiglie nobili Toscane et Umbre, Firenze, stamperia di Francesco Livi, 1673, vol. III, pp. 236 -246. Altre notizie sui Davanzati si trovano raccolte nelle Carte Pucci e Carte Dei del R. Arch. di stato di Firenze e scarsi accenni anche nelle Tavole illustrative di alcune famiglie fiorentine patrisie e popolane di Sebastiano Brigidi in Letture di famiglia, XXXII, 1875, 9-10.

Spogli Ancisa in R. Arch. di Stato di Firenze, AA, 778.

Ib., LL, 373.

4 Ib., LL, 373.

5 Il Libro di Montaperti pubblicato per cura di Cesare Paoli nel vol. IX dei Documenti di Storia Italiana a cura della R. Deputazione di Storia Patria, Firenze, Vieusseux, 1889, pp. 17 e 18. 
estralti dai protocolli di ser Guido Mangiadori che si riferiscono a una domina Guida vidua $f$. et uxor quondam Clari Davanzati $p p$. Sancle Maria supra Arrum e ai figliuoli di costei. 1 Nel primo essa richiede al giudice Rodolfo sibi dari mundualdum ad omnia sua negotia exercenda (Nov. pag. 405); nel secondo, steso immediatamente dopo, è nominata tutrice testamentaria di Martinuccio e Bartolo, Lucia e Lori pupillorum filiorum filiarum q. Clari predicti. $\mathrm{Da}$ entrambi gli atti risulta dunque che Chiaro era già morto nel I 280 e aveva lasciato due maschi (Martinuccio e Bartolo) e due femmine (Lucia e Lori). $\mathrm{Ma}$ ecco in un instrumento del notaio Matteo Biliotti, in data 27 aprile 1304, ricordato come figliuolo del fu Chiaro Davanzati, assieme a Bartolo, anche un Lapo il quale non figura affatto regli atti del I 280.2 Come mai? Il Novati non seppe sciogliere la difficoltà. „亡 lecito supporre, egli si chiede, che Lapo, maggiorenne, fosse per $i$ suoi traffici lontano da Firenze e quindi non in grado di aiutare la madre nell' amministrazione del patrimonio? La cosa non mi par troppo chiara; nè d' altra parte si può pensare che si tratti di una diversa famiglia; perchè il Bartolo ricordato nell' atto del $\mathrm{I}_{304}$ è certamente quel medesimo del quale, fanciullo, fa cenno il documento di ventiquattr' anni innanzi (pag. 407)." Troppo presto il Novati ha scartato quest' ultima ipotesi. Se avesse osservato, come più tardi venne fatto al Torraca, ${ }^{3}$ che l' atto del 1304 dice: Lapus el Bartolus fratres, filii quondam Chiari Davanzati, cives et mercatores forentini, pop. Sancti Frediani, mentre lo sposo di domina Guida è detto del popolo di Santa Maria, si sarebbe facilmente convinto che si tratta per l' appunto di un' altra famiglia e che il Chiaro Davanzati ricordato nel I304 come padre di Lapo e Bartolo è persona ben distinta dall' omonimo morto già nel 1280 e deve identificarsi con l'altro Pavesario ricordato nel Libro di Montaperti (pag. 17, ediz. cit.) col nome di Clarus f. Davanzali Banbakai.

Tanto è vero che, pure volendo dare ragione al Novati, noi non potremmo spiegarci la strana assenza di Lapo dai documenti del $\mathrm{I} 280$, perchè $\mathrm{e}$ infondato il dubbio che in quell' anno egli stesse fuori di Firenze; si deve anzi pensare il contrario se si pon mente ai seguenti fatti.

Nel Libro delia Tavola di Riccomano, si legge: Anno datto Chiarissimo Falchonicri e chonpagni in forini, die XV. giennajo nel 'LXXIIIJ, lib. $D C C C C V \cdot$ avenione seciento sesanta nove fiorini d' oro per soldi ventisette e medalia l' uno, e nove fiorini da dodici. recho Lapo Daranzati.4 Il figliuolo di Chiaro era dunque a Firenze nel 1274;

1 F. Novati, Notizie biografiche di rimatori italiani dei secoli XIII e $X I V$ in Giorn. Stor. della Lett. Ital., vol. V, 1885, pp. 402-407.

2 Cfr. Novati, art. cit., p. 4.

- Per la storia letteraria del sec. XIII in Rass. Crit. della Lett. Ital., vol. X, 1905, p. 118.

- Libro della tavola di Riccomano facopi a cura di C. Vesme in Arch. stor. ital., serie III, vol. XVIII, 1873, p. 14. 
la testimonianza, per quanto interessante, non ha però molto valore, perchè di sei anni anteriore al 1280 e potrebbe benissimo supporsi che nel frattempo egli si allontanasse dalla patria, sennonche altre ne occorrono con data che al 1280 è posteriore. Negli stessi protocolli di ser Guido dei Mangiadori Lapo figura in un instrumento del 1283 qual testimonio per una certa donazione avvenuta 1 ; nel 1284 poi, dal giugno all' agosto, è dei Priori del Comune ${ }^{2}$ ed è indubitato che egli non avrebbe ricoperto quell' ufficio se i traffici lo avessero già condotto a stabilirsi fuori di Firenze.

Privata anche del sussidio di quest' ultima congettura, l' opinione del Novati cade definitivamente e resta accertato che il padre di Lapo e di Bartolo ricordato nell' atto notarile del 27 aprile ${ }_{1304}$ non è il Chiaro Davanzati di cui si fa cenno negli atti del 1280 . Rimane ora a vedere se con la paternità di Lapo non gli sia stata ingiustamente attribuita anche la gloria di poeta.

Che il rimatore debba identificarsi col Chiaro Davanzati del popolo di $\mathrm{S}$. Frediano lo ha dimostrato, non è molto, il Debenedetti nel suo saggio così pregevole sulla Raccolta Giuntina di rime antiche. ${ }^{3}$

\section{Al sonetto famosissimo}

Provedi saggio ad esta visione:^

che Dante da Maiano indirizzò, secondo allora costumavasi, a tutti i fedeli d' Amore, risposero, fra gli altri, l' Alighieri e il Davanzati. Si trattava di una gustosa avventura toccata al poeta; occorre ripeterla? Una donna di bei sembianti, incontrandolo, gli aveva offerto una ghirlanda di fiori; dopo, chi sa mai perchè, il poeta si trovo in dosso una camicia della gentile donatrice e allora, fatto ardito, cominciò a baciarla ed abbracciarla, nè colei si schermiva, anzi l' incoraggiava col sorriso; poi ... ma a questo punto il poeta si accorge di aver detto troppo e si ricorda di un tal giuramento di non parlare; si accorge anche, e probabilmente con infinito suo rammarico, d' essersi espresso troppo chiaramente e rimedia ai due falli col troncare all' improvviso la narrazione e col suggellare il sonetto con un verso oscurissima tanto da págarci dei precedenti di facile intendimento.

Rispose adunque fra gli altri il Davanzati e nel suo sonetto che incomincia

1 R. Arch. di Stato di Firenze, I, 104, f. 75 t.

- Cfr. la Istoria Fiorentina di Marchionne di Coppo Stefani pubblicata dal padre Idelfonso di S. Luigi nelle Delizie degli Eruditi Toscani dell' Affó, Firenze, 1729, vol. VIII, p. 29.

- S. Debenedetti, Nuovi studi sulla Giuntina di rime antiche, Città di Castello. Lapi, IgI2. Per le notizie che specialmente ci interessano, cfr. pp. 15-19.

- Scnetti e canzoni|di diversi|antichi autori toscani| in dieci libri raccolte || Impresso in Firense per li heredi di Philippo di| Giunta nell' anno del Signore | M.D.XXVII. a di VII. | del mese di Luglio c. 140 e il sonetto di risposta del Davanzati si legge immediatamente dopo a c. 141.

Zeitschr. f. rom. Phil. XXXVIIl. 
Amico provveduto a mia intenzione

dice che in realtà dalle rime del Maianese non sa ben trar vera sentenza, ma che tuttavia si rallegra con lui per la bella ventura occorsagli. Orbene, poichè la tenzone avvenne, come appar dimostrato, 1 nel 1283 , è evidente che il poeta non può essere il Chiaro Davanzati del borgo di S. Maria, morto tre anni innanzi. Nè è da credere con lo Zingarelli 2 che il sonetto di risposta a Dante da Maiano sia da attribuirsi a Lapo Saltarelli solo perchè il Valeriani ${ }^{3}$ ce lo riporta come di questi. Di quanta autorità può esser mai, nel nostro caso, il giudizio del Valeriani? E perchè noi dovremmo credere di preferenza a questi che non conobbe alcuna edizione $o$ alcun manoscritto di rime antiche anteriore alla raccolta giuntina e non al Crescimbeni più esatto e più informato di lui o ad altri, tutti concordi coi Giunti nell' assegnare il sonetto

Amico provveduto 2 mia intenzione

al Davanzati 4? In verità la divergenza del Valeriani è da attribuirsi a sua sbadataggine o a sua ignoranza in proposito.

C' è ancora un' altra prova; un' altra tenzone in cui l' Alighieri e il Davanzati si trovano nuovamente uniti, e non più come semplici e casuali compagni di risposta a un terzo rimatore, ma disputanti soli fra loro. Ce ne diede per primo una fuggevole e incompleta notizia Carlo Witte allorchè nei Romanische Studien richiamò $\mathrm{l}$ ' attenzione degli studiosi sul nostro poeta. ${ }^{5}$

Eccone in breve il contenuto. Io amo, dichiara Dante, una bella a cui ho dato tutto il mio volere; ma sono in dubbio se richiederla o no di pari affetto; Chiaro risponde assumendo, notiamolo bene, il tono di persona in la con gli anni e perciò ben esperta delle faccende di questo mondo e consiglia Dante di richieder pure la donna d'amarlo e certamente la troverd pietosa e condiscendente ai suoi prieghi. Il giovanetto Alighieri però non è convinto. Nessun uomo, egli pensa, deve donna pregare

di cosa che pud lei danno tenere (sen: III, $\mathbf{P}_{\text {: }}$ 4)

meglio rimanere col proprio desidero nel cuore, amare tacendo; per tal modo cresce il nostro valore. C' era in lui l' ingenuità del novellino!

1 Cfr. Debenedetti, op. cit., pag. 44.

- N. Zingarelli, Dante, Milano, Vallardi, pag. 7 Io (nota alla pag. 89 del testo).

- Valeriani, Poeti del primo secolo della lingua italiana, Firenze, 1816, vol. II, pag. 437 .

- G. M. Crescimbeni, Dell' istoria della volgar poesia, Venezia, Baseggio, 1730, vol. III, pag. 103. Come di Chiaro Davanzati lo riportano anche l' Occhi, Rime di diversi antichi autori toscani, Venezia, 1740, pag. 35 e il Villarosa, Raccolta di rime antiche toscane, Palermo, Assenzio, 1817, vol. III, pag. 334.

- C. Witte, Chiaro Davanzati in Romanische Studien del Böhmer, 1871, vol. I, pp. II 4-II7. 
Chiaro se ne accorge e, non senza sorriderne, lo riprende paternamente:

se credi per beltate o per sapere

la donna ch' ami sia d' amor sì accesa

ch' ella ti dica si sanza cherere

di ciò ch' '' o detto mi puoi far ripresa

(son. IV, vv. I-4)

concludendo con questi versi per verità un pó oscuri:

chè buona donn' a Dio ben' è demessa,

l' amanza d' huom carnal è di tal planto;

a null' altra lo amor non et disdegno.

(son. IV, vv. 12-I4)

cioè: tranne una buona donna, devota a Dio per la quale l' amore carnale di un uomo è solamente pianto, dolore, tutte le altre non disdegnano le richieste d' amore.1

Sostenuto dall' autorevole giudizio del Barbi, non esito a credere autentica la tenzone 2 ; essa si trova, oltre che nel cod. Marc. IX ital. 191, nel Mgl. VII, 1187. Il primo, miscellaneo del sec. XVI, fu messo assieme da uno studente di legge, Antonio Isidoro Mezzabarba, come risulta da una sua stessa dichiarazione scritta nella seconda guardia del codice ${ }^{3}$; il secondo, anche miscellaneo, di scritture varie, misto di prosa e di versi, per il quinterno che ci interessa è derivazione del Marc. IX, ital. 19 I, (anche qui mi sovviene l' autorità del Barbi) ma non diretta; tra i due dobbiamo supporre un terzo codice finora ignoto a noi.4 Le rime contenute nel quinterno del $\mathrm{Mgl}$. sono le seguenti:

1 Il Bertacchi, primo editore dei sonetti (Le rime di Dante da Maiano ristampate e illustrate da G. B., Bergamo, Istituto Italiano d' arti grafiche, 1896) dichiara circa questi ultimi tre versi: cosi $i$ due codd. ne arrivo ad in. tendere che voglian dire: forse il "bene" debbasi correggere in "tene" ? - A me sembra che si possa evitare di cambiar la lezione manoscritta, cosa sempre pericolosissima, e splegarne il senso come ho spiegato solo che tra il "donna"

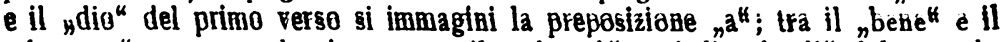
"demessa ${ }^{\mu}$, sempre nel primo verso, il verbo ${ }^{\mathrm{e}^{\mu}}$ e si dia al ${ }^{\mathrm{di}} \mathrm{di}^{\mu}$ del secondo verso il valore di ${ }^{\text {per }}$ "riferito a persona.

M. Barbi, Per una ballata da restituirsi a Dante in Bull. della Soc. Dantesca Ital., 1912, vol. XIX, pp. 6-23. Cfr. anche De Geronimo, Alcune osservasioni sul cod. Marc. It. IX, 19I in Giorn. Stor. della Lett. Ital. I9II, vol. LVII, p. 47 e segg. Il cod. Mgl. VII, I 187 fu una prima volta fuggerolmente ricordato dal Casini: Sopra alcuni manoscritti di rime del secolo XIII in Giorn. Stor. della Lett. Ital., 1884, vol. III, pp. 164 e 191; ne discorre il Barbi nello studio citato a pp. 36-39.

- ${ }_{n}$ Io Antonio Mezzabarba veneto de luna e laltra legge minimo dei scolari ho scritto tutto questo libbro di mia propria mano: nulla mutando overo aggiungendo di quello che io in antiquissimi libbri trovai scritto, ad laudem Dei et gloriosae Virginis, etc. MDIX del mese di maggio". Cfr. A. Della Torre, Aneddoti petrarcheschi in Giornale Dantesco, 1908, vol. XVI, fasc. 3 e 4 , p. 82 e segg.

4 Cfr. Barbi nello studio citato, pp. 38-39. 
1. $30^{\mathrm{b}} \ldots$ La bella stella che 'l tempo misura (C.; ma soltanto l' ultima stanza e il congedo).

2. $30^{2}$ CANZON VJ DI CINO O ... DI ROSSO DOCTORE DI LEGGIE. La somma uirtu di amore acui piacque (continua a c. $16^{2}$ e termina a c. $\left.17^{2}\right)$.

3. $17^{2}$ CANZONA DI DANTE. O caro amico homai conuien chio lachryme.

4. $18^{\text {a }}$ CANZON IJ DI M. GUIDO CAUALCANTI. Io non pensauo che locor gia mai.

5. $19^{2}$ DANTE A CHIARO DAUANZATI. Tri pensieri haggio onde mi uien pensare (S.).

6. $19^{b}$ RISPOSTA PER CHIARO. Per uera experientia di parlare (S.).

7. DANTE A CHIARO. Gia non mi agienza chiaro il domandare (S.).

8. $20^{2}$ CHIARO ADANTE. Se credi per beltate o per sapere (S.).

9. CAN. XV DI DANTE. Al poco giorno et al gran cerchio di ombra.

10. $21^{2}$ M. HONESTO DA BOLOGNA A M. CINO. Si mi e facta nimica la mercede (S.).

I I. $^{\text {b }}$ RISPOSTA DI M. CINO. Messer lo mal che nella mente siede (S.).

12. DI M. CINO. Amor la doglia mia non ha conforto (B.).

13. $22^{2}$ GUIDO NOUELLO DE PULENTA. Ogni dilecto et bene (B.).

14. $22^{\mathrm{b}}$ M. CINO. Poi che suiar non posso gliocchi miei (B.).

15. $23^{2}$ GHIRARDO DE CASTRO FIREN. Guardate in che belta mia donna regna (B).

16. DI M. FRANCCO. PETRARCHA. Sio potessi cantar dolce et soaue (S.).

17. $23^{b}$ IL MEDES(IM)O. Stato fussi io quando la uidi prima (S.).

18. GHUIDO NOUEJLLO. Io sento il sommo bene (B.).

19. $24^{2} \ldots$ Questi mi face una donna guardare (e tutto il resto della canz. di Dante "Voi che intendendo").

20. $25^{2}$ CANZONE TERZA DI DANTE DOUE PARLA DELLA UIRTU ET BELLEZZA DELLA SUA DONNA. Amor che nella mente mi ragiona.

21. $27^{b}$ CANZONE QUARTA DI DANTE DOUE NOBILMENTE PARLA DE LA GENTILEZA ET BEI COSTUMI. Le dolce rime di amore chio solea (la sola $\mathrm{I}^{2}$ stanza).

22. $2^{\text {b }}$ CANZONE DI HYERONIMO BENIUIENI NOBILE POETA ET PATRITIO FIORENTINO.

Amor sotto il cui fren laudace mente.

Per poco che noi fermiamo l' attenzione alla tavola che ho riportata, trascrivendola dall' accurato studio del Barbi più volte oramai citato, 1 ci accorgeremo facilmente che le rime in essa con-

1 Si trova a pp. 36-37. 
tenute sono in gran parte o di autori poco noti o delle meno divulgate se di poeti di maggior grido. Prendiamo ad esempio il Petrarca: i due sonetti di lui riportati (n. 16 e 17) fanno parte l' uno e l' altro delle rime disperse, fuori ogni dubbio assai meno conosciute delle consorelle accolte nel canzoniere. 1 Come mai? Io sospetto che fosse intenzione di chi per primo compilo la raccolta dalla quale direttamente $o$ indirettamente derivarono le loro il Mezzabarba e il trascrittore del ms. Mgl. dar posto in essa per l' appunto a quei componimenti $i$ quali, per una ragione o per l' altra, erano di più rara lettura e perció dei meno noti. Se le cose stanno veramente a questo modo, noi ci possiamo anche spiegare come mai la tenzone fra l'Alighieri e il Davanzati si rinvenga in questi due mss. soltanto e non altrove. Nè la spiegazione è oziosa, perchè è certo che tanto più si sospetta della falsità di un componimento, quanto minore è il numero dei mss. in cui si ritrova.

Ma Dante, è poi proprio l' Alighieri o non piuttosto il suo omonimo maianese? Per il Bertacchi nla struttura, il linguaggio, lo stile, l' intimo sapor di queste rime è tale che non sarà difficile al colto lettore di sentirvi tutta l' aura della maniera arcaica del Maianese senza che occorra dilungarci in minute ricerche ${ }^{2}{ }^{4}$ E un modo assai sbrigativo di far la critica. Quanto siano fallaci le argomentazioni di tal natura, basate soltanto sulla impressione del tutto soggettiva che si riceve dalla lettura di un testo, non è chi non vegga; tanto più, quando si tratta del periodo delle origini di una letteratura in cui una patina di arcaicita uniforma non poche delle produzioni letterarie e rende assai difficile determinare quella che noi chiamiamo originalita dell' autore. Del resto neanche il Bertacchi doveva essere ben convinto di quanto afferma, poichè non sa trovare altri argomenti al suo asserto e taglia corto la questione. Non par dubbio che si tratti proprio dell Alighieri, perchè anche le canzoni sicuramente di lui riportate negli stessi mss. ${ }^{3}$ sono dette semplicemente di Dante, senza aggiunta di cognome. Se i sonetti fossero stati del Maianese, il trascrittore avrebbe avuto cura di avvertircelo per non farci ingenerar confusione. Come si può immaginare che in una stessa raccolta chiamasse promiscuamente Dante e l'Alighieri e il Maianese? Bisogna abituarsi a riconoscer nei copisti più diligenza e discernimento di quel che comunemente non si faccia. E poi Dante era Dante, era lui solo, l' Alighieri, il poeta "che sopra gli altri com' aquila vola", da tutti conosciuto e famosissimo ovunque per cui bastava accennarne il solo nome di battesimo senza pericolo che fosse confuso con altri. Come si

1 Si possono leggere pell' edizione del Solerti (Rime disperse di Francesco Petrarca, Firenze, Sansoni, 1909, pp. 203 e 206) alla cui diligenza però sfuggi di ricordare il presente cod. per il secondo sonetto.

2 G. Bertacchi, ediz. cit. di Dante da Maiano, p. 74. dal Barbi.

- Si confrontino ad esempio i numeri $9,19,20$ e 2 I della tavola riportata 
fa a credere che l' oscuro Maianese di cui la Giuntina non aveva ancor divulgato le rime, avesse tal rinomanza da competere per fama con l'Alighieri?

Determinata così, e a me pare fuori d'ogni dubbio, la personalità storica del rimatore fiorentino, cerchiamo di allacciare in tenue trama le scarse e incomplete notizie che intorno alla vita sua e dei congiunti suoi più prossimi ci rimangono.

L' essersi trovato fra i Pavesari del Comune a Montaperti nel di fatale della battaglia ci farebbe riportare la data della sua nascita almeno al 1240 , se pure non deve ritenersi per certo che nel '6o aveva già passato il quarto lustro d' età come ci indurrebbero a credere alcuni confronti fra il canzoniere di frate Guittone e il suo. Nell' attività poetica di Chiaro Davanzati dobbiamo riconoscere due periodi ben distinti: un primo in cui ormeggia Guittone e ne ripete gli atteggiamenti e $\mathrm{i}$ motivi: un secondo in cui si libera dal modello del maestro, gli si contrappone per alcune idee e segue più spedito per una via sua propria. Il mutamento avviene nel 126 , quando l' Aretino, divenuto frate, negò ogni suo giovanil trasporto amoroso e si mise a predicare la saggezza, la austerità, la virtù con stucchevole pedanteria. 1 Una sua canzone:

$O$ tu di nome Amor, guerra di fatto (Val. I, IV)²

par diretta proprio a ribattere alcune affermazioni che, circa l' amore, il Davanzati aveva cantate in un' altra. Per questi, verace amore ¿ Deo, ma per Guittone:

Peggio che guerra, Amor, uomo te lauda

Tal, perche forte hailo ingegnato tanto,

$\mathrm{Ch}$ ' ello te crede Dio possente e santo.

(Val. I, IV, vv. 16-18)

Per il poeta fiorentino:

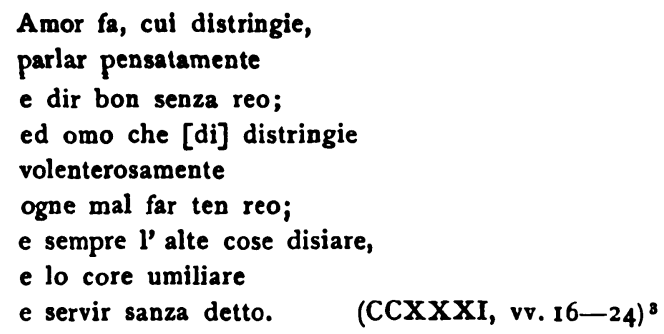

1 Sulla data della conversione di Guittone cfr. A. Pellizzari, La vita e Le opere di Guittone d'Aresso, Pisa, Nistri, 1906. p. 197.

2 Cito, in attesa del secondo volume del Pellegrini, dall' ediz. del Valeriani, Le rime diff. Guittone d'Arezzo, Firenze, Morandi e figlio, 1828 .

3 Mi servo dell' ediz. che del cod. Vat. 3793, unico in cui si trovino raccolte quasi tutte le rime del nostro poeta, ci ha procurato la societa Filologica Romana: Il libro de varie romanze volgari, cod. Vat. 3793, a cura di 
Guittone a sua volta nega e nega che tutto ciò sia vero. Affermano, egli dice, che tu, Amore, fai:

\author{
le vil pro, parlador lo nescente, \\ e lo scarso mettente \\ e leal lo treccante \\ ... e valere 'l selvaggio \\ Ma chi ben sente il contrar vede aperto.
}

(Val. I, IV, vv. 20-24)

Il dissidio tra gli spiriti dell' uno e dell' altro poeta è palese. $E$ allora, quando noi in altro luogo del canzoniere di Chiaro ${ }^{1}$ leggiamo una sua canzone che è tutta un inno di lode a Guittone, dobbiamo dedurne che essa è stata scritta prima che $i$ due rimatori si incaminassero per diversi sentieri, prima cioè del $126 \mathrm{I}$, l' anno in cui, convertitosi Guittone a una fede più rigida e ad una morale più austera, mancò l' accordo fra il pensiero dei due poeti.

Orbene, poteva Chiaro a soli vent' anni aver composto un numero così rilevante di rime da essere già annoverato fra i seguaci della scuola guittoniana, e più ancora, da poter dire di sè:

Valer voria $s^{\prime}$ io mai fu validore o s' unque valsi per saver ben dire!

(CCXLVI, จv. I-2)

Non dico già che sia impossibile, ma è certo più facile immaginarlo di età maggiore; perciò, se la sua presenza a Montaperti ci impedisce di crederlo nato dopo il 1240 , la canzone in lode di Guittone ci consiglia a risalire ancora di qualche anno addietro. Il poeta avrà dunque veduto la luce tra il 1230 e il 1240 .

Certamente fu a Pisa; ne è testimonianza il suo stesso canzoniere ${ }^{2}$ e quivi il cuor suo conobbe le dolcezze dell' amore. Costretto qualche tempo dopo a ritornare in patria, la sua mente riandava con accorata malinconia ai bei dì passati nella

$$
\text { gientile terra sovra ogni altra pisana }
$$

(CCLVI, v. 4I)

e allora lo assalivano la nostalgia e la tristezza delle cose perdute.

F. Egidi, S. Satta e G. B. Festa, Roma, Loescher, 1902-1908, bene inteso, dividendo le parole, punteggiando e rammodernando la grafia.

1 Cod. Vat. 3793, CCXLVI.

- Cod. Vat. 3793, CCLIV, CCLVI e CCLVIr. Sono tre canzoni di lontananza nelle quali si rimpiange la bella lasciata a Pisa; nella CCLVII per vero si parla di una lontana riviera, non di Pisa, ma non può dubitarsi che l'accenno vada fatto a quella città se si pon mente ai versi $40-41$ della canzone precedente: dolze riviera gaia ed amorosa | diletto sovra tute la pisana. 
La mia spene è divisa, non gia di bene amare, ma di paese ch' agio prolungato.

La mente e 'l core è 'm Pisa: tuto lo mio pensare

davanti al' avenente in quello lato

(CCLIV, vv. 40-45)

In quale anno? Io credo che il poeta lasciò Firenze per Pisa nel 1 267, quando i Guelfi di Firenze, baldanzosi per le vittorie di Carlo D'Angiò sugli Svevi, offrirono a questi per dieci anni la signoria della città; ci è noto infatti come molti ghibellini in quella occasione esularono; e che Chiaro parteggiasse per costoro appare evidente dalle sue rime politiche. 1

Le altre poche notizie che di lui si son potute rintracciare furono gid adunate dal Debenedetti e a me non resta che riferirle con qualche piccola aggiunta, rimandando a questi per la fonte da cui sono state tolte. 2

Nel 1294 il Davanzati fu capitano di Or San Michele da calen di febbraio a calen di giugno; il 23 settembre del $1302 \mathrm{fu}$ da Johannes de Calona, arcidiacono di Gent, nominato suo procuratore assieme a Petrus Telli, della società degli Spini e in tale qualità compare in un atto dell' agosto del 1303 la quale data è l' ultima che conosciamo della sua vita. Il 27 aprile 1304 era già morto lasciando, oltre $\mathrm{i}$ due figli Lapo e Bartolo di lui fa cenno il documento pubblicato dal Novati, una figliuola, Bionda, sposa a Banco di Jacopo, già vedova nel 1317 e ancor viva il 9 luglio I32I.

Di Lapo s' è detto che nel 1274 recò nove fiorini a Riccomano di Jacopo; qui aggiungo come nel 1308, assieme a Bartolo di Benci di Ristoro, ai fratelli di questi e a più altri, fu costretto a cedere al Comune le sue case e terreni presso il palazzo del Potestà per la costruzione del Palazzo del Vicario e ne ebbe in cambio lire 6675 oltre certi terreni e botteghe e case nel borgo di S. Lucia $e$ in quella di S. Stefano ${ }^{3}$; nel 1313 infine è compreso nel bando di Enrico VII. contro tutti i ribelli di Toscana 1 e altro non sappiamo di lui; del fratello Bartolo ricorre il nome ancora nel 1317 qual testimonio di certa donazione fatta dagli eredi dei Peruzzi.5

1 Rimando in proposito al mio opuscolo, La poesia politica di Chiaro Davansati, Ravenna, tip. Lavagna, I913.

Debenedetti, op. cit., pp. 15-19.

- Cfr. Affo, cit., vol. V, p. 139 che ricava la notizia dal Libro delle Riformagioni ove è data su testimonianza di Bonaguida del Fabro Tolosini e di Marco del fu Jacopo dell' Asino.

- Cfr. Affo, cit., vol. V, p. 131.

s Cfr. Affo, cit., vol. XVI, p. 516. 
Ricerche più diligenti di altri studiosi potranno in avrenire portare nuovi contributi che valgano a meglio lumeggiare la vita - ahimè, ancora così avvolta nelle tenebre! - del singolare poeta duecentesco. Per me son pago se con queste mie osservazioni sono riuscito a determinarne la sua personalità storica e a raggtuppare intorno ad essa quelle poche notizie che per ventura si son salvate dal naufragio del tempo.

RUgGero Palmieri. 